\title{
Asymmetric wireless power transfer systems using coupled DGS resonators
}

\author{
Sherif Hekal ${ }^{1,2 a)}$, Adel B. Abdel-Rahman ${ }^{2,3}$, Ahmed Allam ${ }^{2}$, \\ Hongting Jia ${ }^{1}$, Adel Barakat ${ }^{1,4}$, and Ramesh K. Pokharel ${ }^{1 b}$ \\ ${ }^{1}$ Center for Japan-Egypt Cooperation in Science and Technology, \\ Kyushu University, Motooka 744, Nishi-ku, Fukuoka 819-0395, Japan \\ ${ }^{2}$ Electronics Communications Engineering Department, Egypt-Japan University of \\ Science and Technology, New Borg El-arab City 21934, Alexandria, Egypt \\ ${ }^{3}$ Electrical Engineering Department, South Valley University, Qena 83523, Egypt \\ ${ }^{4}$ Microstrip Department, Electronics Research Institute, Giza 12622, Egypt \\ a)sherif.hekal@ejust.edu.eg \\ b)pokharel@ed.kyushu-u.ac.jp
}

Abstract: This paper presents a new design for wireless power transfer (WPT) systems using asymmetric structures for the transmitter (TX) and the receiver (RX), and the TX/RX are constructed using spiral-strips defected ground structure (DGS) resonators. The proposed spiral-strips DGS resonator overcome the problem of low self-inductance that encountered by H-shape DGS resonator in [1, 2], so that the proposed WPT system that employs the proposed spiral-strips DGS resonators has better efficiency and higher power transmission distance. Design methodology of the proposed WPT system are formulated and fabricated. The measurement results show a WPT efficiency of $78 \%$ at a transmission distance of $40 \mathrm{~mm}$ with the TX and RX areas of $50 \times 50 \mathrm{~mm}^{2}$ and $30 \times 30 \mathrm{~mm}^{2}$, respectively.

Keywords: defected ground structures, EM resonant coupling, wireless power transfer

Classification: Microwave and millimeter-wave devices, circuits, and modules

\section{References}

[1] S. Hekal and A. Abdel-Rahman: "New compact design for short range wireless power transmission at $1 \mathrm{GHz}$ using H-slot resonators," EUCAP Proc. (2015) 1 (ISSN 2164-3342).

[2] S. Hekal, et al:: "Strong resonant coupling for short-range wireless power transfer applications using defected ground structures," WPTC Proc. (2015) 1 (DOI: 10.1109/WPT.2015.7140185).

[3] F. Jolani, et al.: "A planar magnetically coupled resonant wireless power transfer system using printed spiral coils," IEEE Antennas Wireless Propag. Lett. 13 (2014) 1648 (DOI: 10.1109/LAWP.2014.2349481).

[4] F. Jolani, et al.: "Enhanced planar wireless power transfer using strongly coupled magnetic resonance,” IET Electron. Lett. 51 (2015) 173 (DOI: 10. 1049/el.2014.4104). 
[5] M. K. Mandal and S. Sanyal: "A novel defected ground structure for planar circuits," IEEE Microw. Compon. Lett. 16 (2006) 93 (DOI: 10.1109/LMWC. 2005.863192).

[6] N. C. Karmakar, et al.: "Quasi-static modeling of defected ground structure," IEEE Trans. Microw. Theory Techn. 54 (2006) 2160 (DOI: 10.1109/TMTT. 2006.873633).

[7] L. H. Weng, et al:: "An overview on defected ground structure," Progress In Electromagnetics Research B 7 (2008) 173 (DOI: 10.2528/PIERB08031401).

[8] S. U. Rehman, et al.: "Compact bandstop filter using defected ground structure (DGS)," SIECPC Proc. (2011) 1 (DOI: 10.1109/SIECPC.2011.5876972).

[9] A. Abdel-Rahman, et al.: "Compact stub type microstrip bandpass filter using defected ground plane," IEEE Microw. Compon. Lett. 14 (2004) 136 (DOI: 10. 1109/LMWC.2003.821503).

[10] A. Abdel-Rahman, et al.: "Compact bandpass filters using defected ground structure (DGS) coupled resonators," IEEE MTT-S International Microwave Symposium Digest 1 (2005) 1479 (ISSN 0149-645X) (DOI: 10.1109/ MWSYM.2005.1516971).

[11] J. Lee, et al:: "Wireless power transfer system adaptive to change in coil separation," IEEE Trans. Antennas Propag. 62 (2014) 889 (DOI: 10.1109/TAP. 2013.2290795).

[12] U. M. Jow and M. Ghovanloo: "Design and optimization of printed spiral coils for efficient transcutaneous inductive power transmission," IEEE Trans. Biomed. Circuits Syst. 1 (2007) 193 (DOI: 10.1109/TBCAS.2007.913130).

[13] T. Imura and Y. Hori: "Maximizing air gap and efficiency of magnetic resonant coupling for wireless power transfer using equivalent circuit and Neumann formula," IEEE Trans. Ind. Electron. 58 (2011) 4746 (DOI: 10.1109/TIE.2011. 2112317).

[14] S. Raju, et al.: "Modeling of mutual coupling between planar inductors in wireless power applications," IEEE Trans. Power Electron. 29 (2014) 481 (DOI: 10.1109/TPEL.2013.2253334).

\section{Introduction}

High efficiency and compact wireless power transfer (WPT) systems are tremendously required for the battery-less functioning of wireless sensors such as biomedical implants, buried sensors and so on. One of the common methods for such WPT systems is using the strongly coupled printed spirals [3, 4]. In strong resonant coupling WPT system, the inductive feeding was employed between driving/load loop and the transmitting (TX)/receiving (RX) resonator; where the driving/load loop realizes the input/output impedance matching [3, 4]. On the other hand, the inductive feeding employed in [3] has the driving/load loop on the same plane to the TX/RX resonator because it is inherent to achieve tight coupling to guarantee the maximum power transfer. Also, the inductive feeding [3] requires a large size of the driving/load loops which may increase the resistive paths of the driving/load loops and thus decrease the external quality (Q-) factor of the resonators. Besides, within a given size of a WPT system, the driving/load loop size limits the area where we cannot optimize the TX/RX resonators' parameters. These parameters include the track width $\left(W_{\mathrm{t}, \mathrm{i}}\right)$ and separation $\left(s_{\mathrm{i}}\right)$, and the number of turns $\left(N_{\mathrm{i}}\right)$ to realize a high unloaded Q-factor. To overcome these problems, the authors of this letter have proposed quasi-lumped elements based on defected 
ground structures (DGS) for wireless power transfer for the first time in $[1,2]$. Etching a DGS on the opposite side of a microstrip feeding line realizes a bandstop filter (BPF) characteristics $[5,6,7,8]$. Also, the DGSs exhibit a bandpass filter (BPF) characteristics by introducing a discontinuity in the feeding microstrip line above the DGS's slots and adding some stubs for matching $[9,10]$. In the same manner, when two DGS resonators are coupled back to back, it results in a bandpass characteristic, and power is transferred from the source to load through the DGS resonators $[1,2]$. In our proposed DGS-WPT system, we use electrical coupling for feeding. Thus, the limitations of the feeding loop is avoided and a higher achievable unloaded Q-factor is possible unlikely in the design proposed in $[3,4]$.

However, the mechanism of power transfer using DGS resonators has not been investigated yet thoroughly. Therefore, in the first part of this letter, mechanism of power transfer using two coupled bandstop filter (BSF) resonators realized by DGS was illustrated. In the second part, we propose the application of the coupled DGS to design a highly efficient and compact asymmetric WPT system. Besides, we demonstrate its successfulness by comparing the design, EM simulation, and experimental results. The electromagnetic (EM) and circuit simulations in this letter are performed using the Ansoft's High Frequency Structure Simulator (HFSS) and Keysight's Advanced Design system (ADS), respectively.

\section{Development of the proposed WPT system using coupled DGS resonators}

One of the first applications of DGS resonators were illustrated by the authors [1] where two H-shape DGS resonators were coupled back to back to make a WPT system, and power is transferred at a distance of $3.5 \mathrm{~mm}$ only. First, reasons for low power transfer distance was investigated and spiral strips DGS was proposed in this letter to mitigate the problems in H-shape DGS resonators that encountered in $[1,2]$.

\subsection{Proposed spiral-strips DGS}

Fig. 1(a) illustrates the H-shape DGS and its modified versions (the semi H-shape, and the spiral-strips DGS) and EM simulated current elongated path, where there are two parallel current paths in H-shape DGS. This causes the low self-inductance of the resonator which results in the lower efficiency at a larger distance $[1,2]$. First, this problem is overcome by constructing a single current path only like in a semi H-shape DGS resonator and the value of inductance is further improved as in spiral-strips DGS resonator. Fig. 1(b) shows the computed inductance of the three DGS resonators, where the elongated current path in the spiral-strips DGS provides twice the self-inductance that of the semi H-shape and four times that of the H-shape using only half the area of H-shape DGS. Thus, the spiral-strips DGS provides the highest Q-factor among the others, and they are employed in this work. 


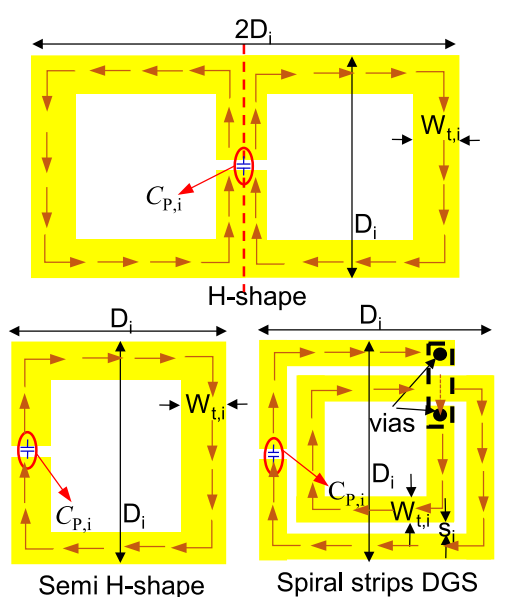

(a)

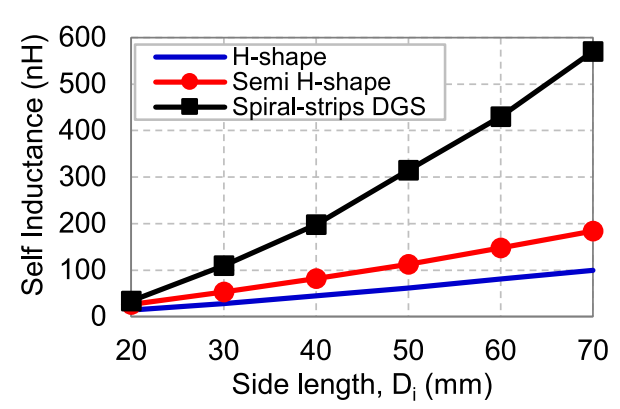

(b)

Fig. 1. Comparison between three different shapes of DGS (H-shape, semi H-shape, Spiral-strips DGS) (a) Current distribution. (b) Computed self-inductance.

\subsection{Proposed WPT system}

In this section, we explain the mechanism and systematic approach to convert a DGS bandstop resonator to a bandpass filter (BPF). Etching a DGS on the opposite side of a microstrip feeding line realizes a stopband notches as shown in Fig. 2. In Fig. 2, we present the model and EM simulation results of the scattering (S-) parameters $\left(\left|\mathrm{S}_{11}\right|\right.$ and $\left.\left|\mathrm{S}_{21}\right|\right)$. First, we design the DGS resonator on Rogers substrate (RO5880) with permittivity $\varepsilon_{\mathrm{r}}=2.2$, thickness $T_{\text {sub }}=0.5 \mathrm{~mm}$, and metal thickness $t=18 \mu \mathrm{m}$, where the top layer is a $50 \Omega$ microstrip line of width $W_{\mathrm{f}, \mathrm{i}}$. The bottom layer is the spiral-strips DGS with a number of turns, $N_{\mathrm{i}}$, outer diameter, $D_{\mathrm{i}}$, track (strip) width, $W_{\mathrm{t}, \mathrm{i}}$, and a separation between the strips, $s_{\mathrm{i}}$. The equivalent $R L C$ values of the proposed spiral-strips DGS, shown in Fig. 2(a), (b), are extracted by Eq. (1)-(3) [7] from its EM band reject response. Where $\omega_{0}$ is the center angular frequency of the stopband, $\omega_{\mathrm{c}}$ is the $3 \mathrm{~dB}$ cutoff angular frequency taken from the $\mathrm{S}_{21}$ curve (shown in Fig. 2(c)), and $\mathrm{Z}_{0}=50 \Omega$.

$$
\begin{gathered}
\mathrm{C}_{\mathrm{P}, \mathrm{i}}=\frac{\omega_{c}}{2 Z_{0}\left(\omega_{0}^{2}-\omega_{C}^{2}\right)} \\
\mathrm{L}_{\mathrm{P}, \mathrm{i}}=\frac{1}{\omega_{0}^{2} C_{P}} \\
R_{i}=\frac{2 Z_{0}}{\sqrt{\frac{1}{\left|S_{11}(\omega)\right|^{2}}-\left(2 Z_{0}\left(\omega C_{P, i}-\frac{1}{\omega L_{P, i}}\right)\right)^{2}}-1}
\end{gathered}
$$

As we previously mentioned in the introduction, a BPF characteristics can be realized by introducing a discontinuity in the feeding microstrip line above the DGS's slots and adding some stubs for impedance matching. Similarly by adding an additional DGS $[1,2]$, the power is coupled between the two DGSs as can be interpreted from Fig. 3(a), (b). In this proposed DGS-WPT system, we use electrical coupling for feeding. Thus, the limitations of the feeding/load loops are avoided, and a higher achievable unloaded Q-factor of 400 is possible unlikely in the design proposed in [3] without/with auxiliary strips (the unloaded Q factors: 


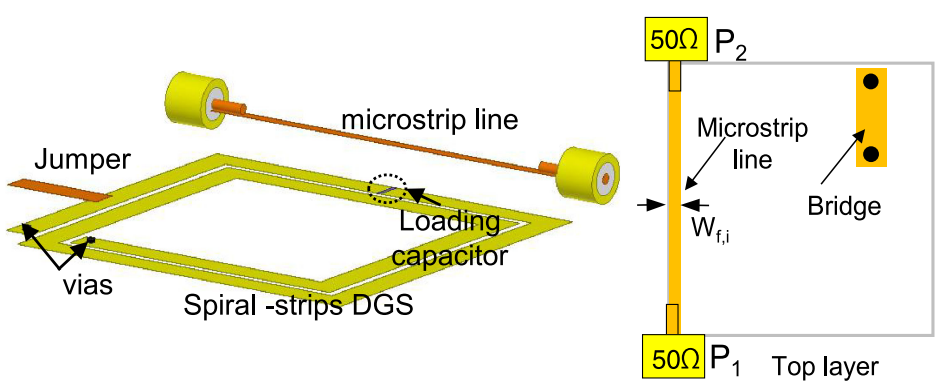

(a)

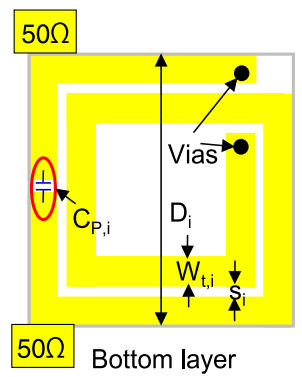

(b)

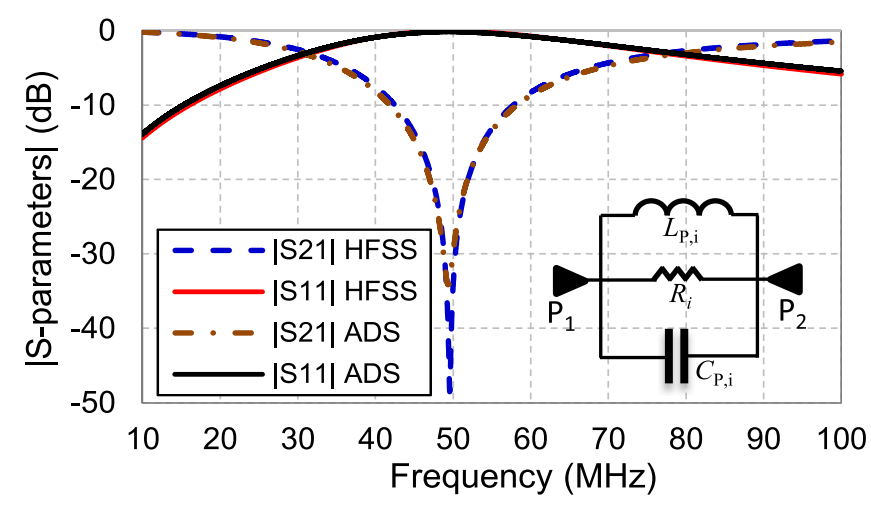

(c)

Fig. 2. The proposed spiral-strips DGS resonator working as BSF (a) 3-D view (b) PCB layout. (c) EM and circuit simulations of $\left|\mathrm{S}_{11}\right|$ and $\left|\mathrm{S}_{21}\right|$.

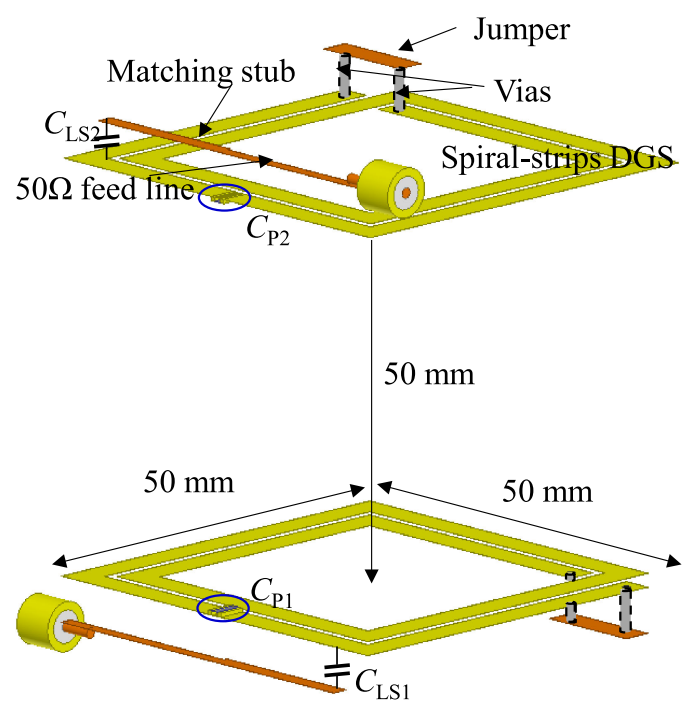

(a)

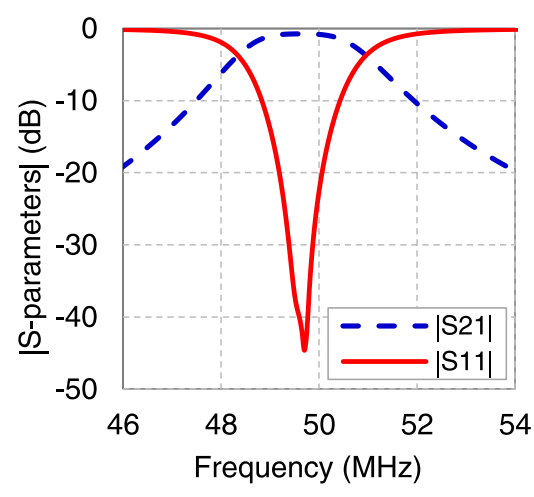

(b)
Fig. 3. (a) Model of the proposed DGS-WPT system, and (b) EM simulated $\left|\mathrm{S}_{11}\right| \&\left|\mathrm{~S}_{21}\right|$.

274, and 328), respectively. Simultaneously, the DGS resonator is fed by a microstrip feeding line loaded by an additional capacitor $\left(C_{\mathrm{LS}, \mathrm{i}}\right)$. This capacitor value optimizes the external Q-factor for high-efficiency WPT system. Fig. 4(a) shows the 2-D PCB layout of the optimized TX/RX resonators for our proposed DGSWPT system. The top layer is a $50 \Omega$ feeding line of length $\left(L_{\mathrm{f}, \mathrm{i}}\right)$ and width $\left(W_{\mathrm{f}, \mathrm{i}}\right)$ 
followed by a stub of length $\left(L_{\mathrm{st}, \mathrm{i}}\right)$ and width $\left(W_{\mathrm{st}, \mathrm{i}}\right)$. The stub is represented by the parallel plate capacitance $\left(C_{\mathrm{st}, \mathrm{i}}\right)$. The tuning capacitor $\left(C_{\mathrm{P}, \mathrm{i}}\right)$ is a chip capacitor to adjust the resonance and minimize the design area. The capacitances $C_{\mathrm{LS}, \mathrm{i}}$ and $C_{\mathrm{st}, \mathrm{i}}$ are used for impedance matching; the impedance matching capacitor (IM cap) $C_{\mathrm{LS}, \mathrm{i}}$ is a chip capacitor connected between the top and the bottom layers, parallel with the capacitance $C_{\mathrm{st}, \mathrm{i}}$ of the stub.

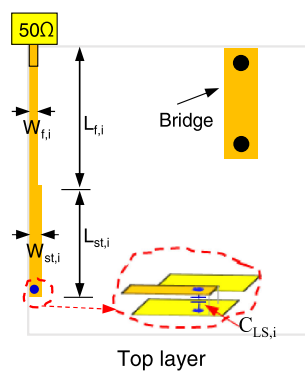

(a)

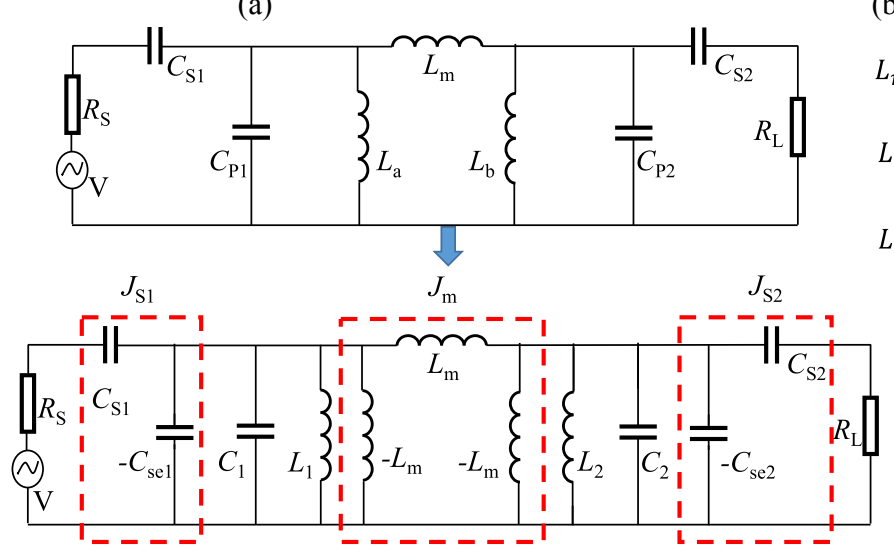

(c)

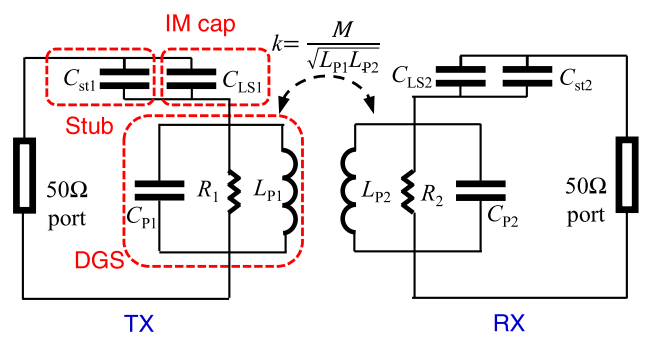

(b)

$L_{m}=\frac{L_{P 1} L_{P 2}-M^{2}}{M}$
$L_{a}=\frac{L_{P 1} L_{P 2}-M^{2}}{L_{P 2}-M}$
$L_{b}=\frac{L_{P 1} L_{P 2}-M^{2}}{L_{P 1}-M}$ $C_{\mathrm{P} 1}=C_{1}-C_{\mathrm{se} 1}$ $C_{\mathrm{P} 2}=C_{2}-C_{\mathrm{se} 2}$ $L_{1}=L_{\mathrm{a}} / / L_{\mathrm{m}}$ $L_{2}=L_{\mathrm{b}} / / L_{\mathrm{m}}$

Fig. 4. (a) PCB layout of the realized TX/RX structure. (b) The equivalent circuit of the proposed WPT system. (c) Analysis of the equivalent circuit using J-inverters [11].

The responses in Fig. 2(c) and Fig. 3(b) have been achieved using the design parameters $\left(N_{1}=2, W_{\mathrm{f} 1}=1.55 \mathrm{~mm}, D_{1}=50 \mathrm{~mm}, W_{\mathrm{t} 1}=3.5 \mathrm{~mm}\right.$, and $\left.s_{1}=1 \mathrm{~mm}\right)$ that give $L_{\mathrm{P} 1}=315 \mathrm{nH}$ and $R_{1}=40 \mathrm{~K} \Omega$, and $C_{\mathrm{P} 1}=33 \mathrm{pF}$. The explanation of the values' selection will be discussed in details in Section 3. Also, the equivalent circuit model of the optimized DGS-WPT system is shown in Fig. 4(b), and its detailed analysis using J-(admittance) inverters is illustrated in Fig. 4(c) to simplify the proposed design method from [11]. According to the simplified Neumann's formulae presented in $[12,13]$, the transmission distance $(h)$ is a function of the diameter $\left(D_{\mathrm{i}}\right)$ and a number of turns $\left(N_{\mathrm{i}}\right)$ of the TX/RX coils. Furthermore, the maximum WPT efficiency is achieved through an optimization of the unloaded Qfactor of individual resonators, mutual coupling, and impedance matching, where the WPT efficiency can be maximized to give $\eta_{\text {opt }}=U^{2} /\left(1+\sqrt{\left(1+U^{2}\right)}\right)^{2}, U=$ $k \sqrt{Q_{1} Q_{2}}$ [12]. The mutual inductance $(M)$ and the coupling coefficient $(k)$ are calculated by Eq. (4) and Eq. (5), respectively [14], where $N_{1}$ and $N_{2}$ are the numbers of turns of the TX and the RX resonators, respectively. 


$$
\begin{gathered}
M=\left(\frac{4}{\pi}\right) \sum_{n=1}^{2 n=N_{1}} \sum_{p=1}^{p=N_{2}} M_{n p} \text { Henry } \\
M_{n p}=\frac{\mu_{0} \pi a_{n}^{2} b_{p}^{2}}{2\left(a_{n}^{2}+b_{p}^{2}+h^{2}\right)^{3 / 2}}\left(1+\frac{15}{32} \gamma_{n p}^{2}+\frac{315}{1024} \gamma_{n p}^{4}\right) \\
a_{n}=\frac{D_{1}}{2}-(n-1)\left(W_{t 1}+s_{1}\right)-\frac{W_{t 1}}{2}, \quad b_{p}=\frac{D_{2}}{2}-(p-1)\left(W_{t 2}+s_{2}\right)-\frac{W_{t 2}}{2}, \\
\gamma_{n p}=\frac{2 a_{n} b_{p}}{\left(a_{n}^{2}+b_{p}^{2}+h^{2}\right)} \\
K=\frac{M}{\sqrt{L_{P 1} L_{P 2}}}
\end{gathered}
$$

\section{Design procedure of the proposed WPT system}

Fig. 5 presents the proposed applications using the proposed asymmetric WPT system. For wireless charging of portable handsets, the TX is built in the charging pad to be hidden under a desk, or inside walls of transportation vehicles and the RX is embedded in the portable handset.
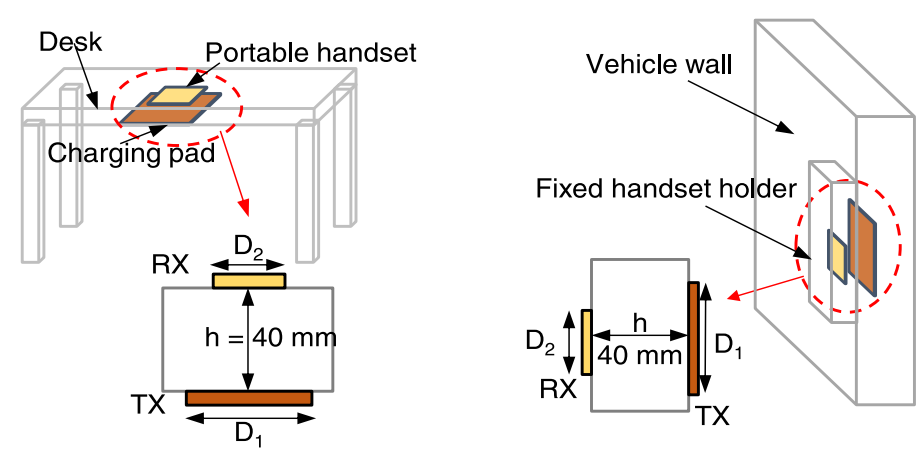

Fig. 5. The proposed applications for wireless charging of mobile handsets.

We selected $f_{0}=50 \mathrm{MHz}$ as the operating frequency for the proposed WPT systems. Considering the size constraints of the intended applications, we can summarize the design steps as the following:

1. Define the required RX area and the necessary transmission distance.

2. For the maximum WPT efficiency according to $[12,13]$,

a. In the symmetric systems, we need $D_{1}=D_{2}=h$; where $D_{1}$ and $D_{2}$ are the outer diameters of both the TX and RX structures, respectively, and $h$ is the transmission distance.

b. In the asymmetric systems, we need $\sqrt{G M A}=h$, where $G M A$ is the geometric mean area of the TX and RX structures, $G M A=$

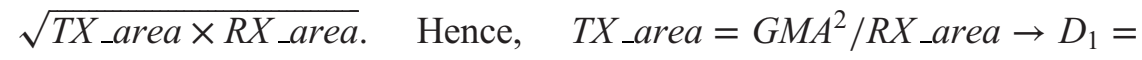
$h^{2} / D_{2}$.

3. Extract the optimum design dimensions $\left(W_{\mathrm{t}, \mathrm{i}}, s_{\mathrm{i}}\right.$, and $\left.N_{\mathrm{i}}\right)$ for both the TX and $\mathrm{RX}$ structures that achieve the highest $U$-factor $\left(U=k \sqrt{Q_{1} Q_{2}}\right)$ using the study shown in Fig. 6, where $k$ is calculated by Eq. (5) and the unloaded Q-factor is calculated by $\mathrm{Q}_{\mathrm{i}}=R_{\mathrm{i}} / 2 \pi f_{0} L_{\mathrm{P}, \mathrm{i}}$. 
4. By substitution with the design parameters defined and calculated using the steps 1-3 in Eq. (1)-(5), we can get the values $L_{\mathrm{P} 1}, L_{\mathrm{P} 2}, R_{1}, R_{2}, M$, and $k$.

5. Apply the Analytic design method in [11] (by substitution in Eq. (2), (6), (5), (1), and (3), respectively, in [11]) to get the remaining circuit parameters $C_{\mathrm{P} 1}$ and $C_{\mathrm{P} 2}$ and the values of $C_{\mathrm{S} 1}$ and $C_{\mathrm{S} 2}$ that are realized in our circuit model by $C_{\mathrm{S} 1}=C_{\mathrm{st} 1}+C_{\mathrm{LS} 1}, C_{\mathrm{S} 2}=C_{\mathrm{st} 2}+C_{\mathrm{LS} 2}$. This design method depends on achieving the perfect impedance matching of the system through satisfying the condition $J_{\mathrm{S} 1} J_{\mathrm{S} 2} R=J_{\mathrm{m}}$ (Eq. (5) [11]), where $R=R_{\mathrm{S}}=R_{\mathrm{L}}=50 \Omega$.

6. Using $L_{\mathrm{st}, \mathrm{i}}$ and $W_{\mathrm{st}, \mathrm{i}}$, the parallel plate capacitance $C_{\mathrm{st}, \mathrm{i}}$ can be easily calculated and so, the required chip capacitor can be calculated from $C_{\mathrm{LS}, \mathrm{i}}=C_{\mathrm{S}, \mathrm{i}}-C_{\mathrm{st}, \mathrm{i}}$.

7. Finally, the design parameters of the proposed symmetric and asymmetric WPT systems are fine-tuned using HFSS for further efficiency enhancement and final optimization before fabrication.

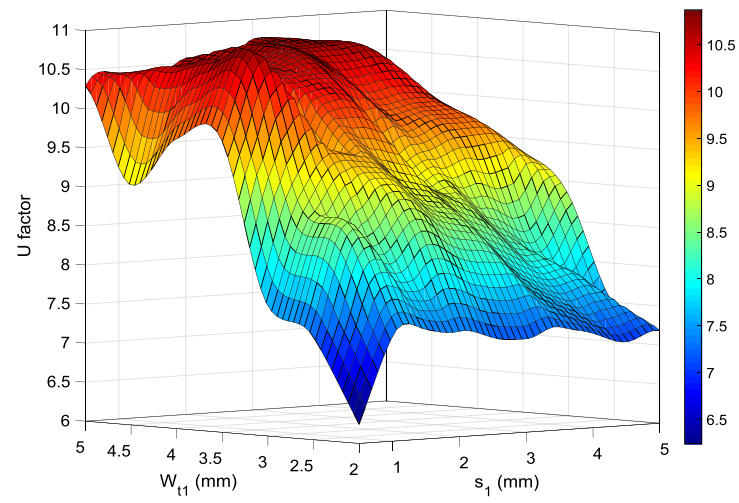

Fig. 6. Investigation of the computed $U$-factor of the coupled resonators versus the width $W_{\mathrm{t}, \mathrm{i}}$ and separation $s_{\mathrm{i}}$ for the proposed symmetric WPT systems $\left(50 \times 50 \mathrm{~mm}^{2}\right)$.

Fig. 6 presents a study on the symmetric WPT system $\left(50 \times 50 \mathrm{~mm}^{2}\right)$ that illustrates the effect of the strip width, $W_{\mathrm{t} 1}$, and the separation, $s_{1}$, variations on the values of the $U$-factor using $N_{1}=2$. We have performed this study also for $\mathrm{N}_{1}=3$, 4 , 5, etc. and found that the highest U-factor is achieved at $N_{1}=2$. Likewise, a study has been performed on the symmetric WPT system $\left(30 \times 30 \mathrm{~mm}^{2}\right)$ and the asymmetric system. These studies have determined the values of $N_{1}=N_{2}=2$, $W_{\mathrm{t} 1}=3.5 \mathrm{~mm}, W_{\mathrm{t} 2}=2.5 \mathrm{~mm}$ and $s_{1}=s_{2}=1 \mathrm{~mm}$ for the highest value of the $U$-factor. Table I details the final design parameters and the equivalent $R L C$ values for the proposed asymmetric WPT system. Fig. 7 shows a glance of the EM simulations for the asymmetric WPT system by showing the magnetic field distribution at different phases $0^{\circ}-180^{\circ}$.

Table I. Optimized design parameters and Equivalent circuit $R L C$ values

\begin{tabular}{|c|c|c|c|c|c|c|c|c|c|c|c|c|c|c|}
\hline & \multicolumn{7}{|c|}{ Design dimensions (mm) } & \multicolumn{7}{|c|}{$R L C$ values: $L, M(\mathrm{nH}), R(\mathrm{~K} \Omega)$, and $C(\mathrm{pF})$} \\
\hline & $L_{\mathrm{f}, \mathrm{i}}$ & $W_{\mathrm{f}, \mathrm{i}}$ & $L_{\mathrm{st}, \mathrm{i}}$ & $W_{\mathrm{st}, \mathrm{i}}$ & $D_{\mathrm{i}}$ & $W_{\mathrm{t}, \mathrm{i}}$ & $S_{\mathrm{i}}$ & $L_{\mathrm{P}, \mathrm{i}}$ & $R_{\mathrm{i}}$ & $C_{\mathrm{P}, \mathrm{i}}$ & $C_{\mathrm{st}, \mathrm{i}}$ & $C_{\mathrm{LS}, \mathrm{i}}$ & $M$ & $k$ \\
\hline $\mathrm{TX}, \mathrm{i}=1$ & 25 & 1.55 & 20 & 2.05 & 50 & 3.5 & 1 & 315 & 40 & 24 & 2 & 7 & 3 & \\
\hline $\mathrm{RX}, \mathrm{i}=2$ & 15 & 1.55 & 11 & 2.15 & 30 & 2.5 & 1 & 150 & 10 & 55 & 1.5 & 11 & 0.5 & 0.04 \\
\hline
\end{tabular}



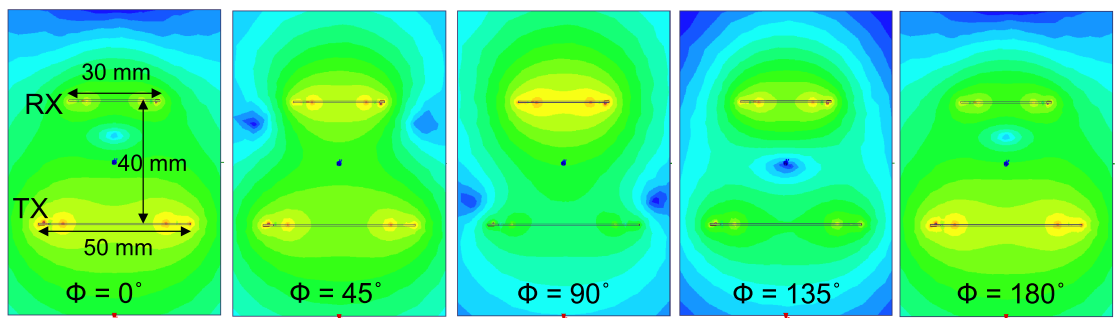

Fig. 7. Magnetic field distribution of the asymmetric WPT system at phases $\Phi=0^{\circ}, 45^{\circ}, 90^{\circ}, 135^{\circ}$, and $180^{\circ}$.

\section{Fabrication and measurements}

Fig. 8 shows the measurement setup for the fabricated WPT systems (symmetric, and asymmetric) using Vector Network Analyzer (Agilent N5222A). Fig. 9(a)-(c) present the simulated and the measured S-parameters $\left|S_{11}\right|$ and $\left|S_{21}\right|$ for the proposed WPT systems, also $\left|S_{22}\right|$ for the asymmetric WPT systems. As shown, the symmetric and asymmetric WPT systems operate at $49.5 \mathrm{MHz}$, and the measured results are in good agreement with the simulated results. The frequency shift between the measured and the simulated |S-parameters| is due to the tolerance in the used chip capacitors values $\left(C_{\mathrm{P}, \mathrm{i}}\right.$ and $\left.C_{\mathrm{LS}, \mathrm{i}}\right)$.
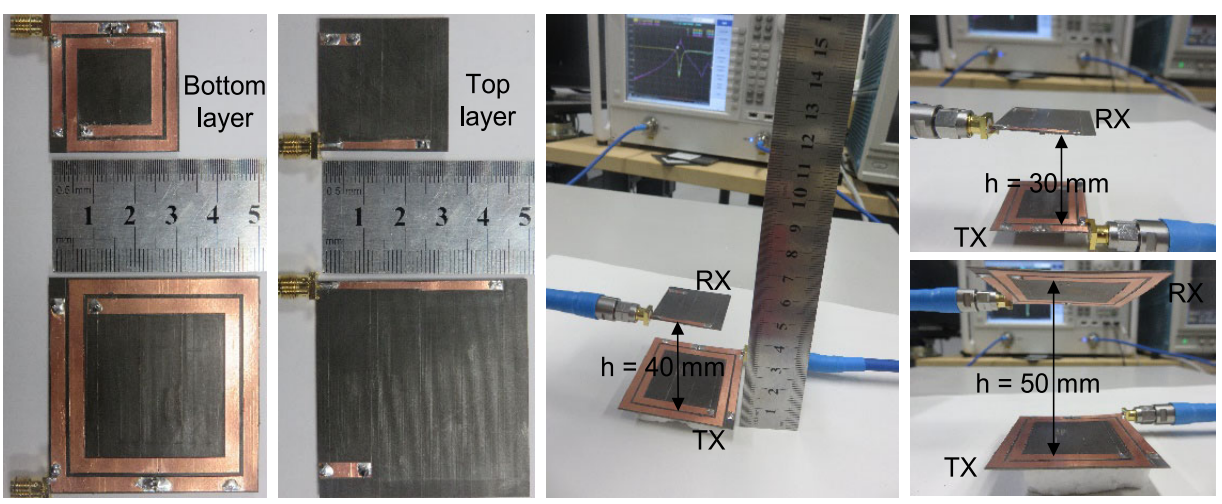

Fig. 8. Measurement setup of the fabricated WPT systems (Symmetric $50 \times 50 \mathrm{~mm}^{2}$, Symmetric $30 \times 30 \mathrm{~mm}^{2}$, and Asymmetric TX $=$ $50 \times 50 \mathrm{~mm}^{2}, \mathrm{RX}=30 \times 30 \mathrm{~mm}^{2}$ ).

The WPT efficiency is calculated using the formula $\eta=\left|S_{21}\right|^{2}$. From Fig. 9(d), the symmetric WPT system $\left(\mathrm{TX}=\mathrm{RX}=50 \times 50 \mathrm{~mm}^{2}\right)$ achieves a maximum efficiency of $84 \%$ at transmission distance $h=50 \mathrm{~mm}$, which is comparable to the value recently obtained in [4], but without using complex stacked substrates. The asymmetric WPT system has achieved a measured WPT efficiency of $78 \%$ at $h=40 \mathrm{~mm}$. The larger area of the TX provides high $U$-factor that enables the asymmetric WPT system to provide a better efficiency at a longer transmission distance than the symmetric case $\left(\mathrm{TX}=\mathrm{RX}=30 \times 30 \mathrm{~mm}^{2}\right)$. Table II presents a comparison between the proposed WPT systems (symmetric and asymmetric) and the recent published planar WPT systems regarding the TX/RX areas, maximum transmission distance, and the measured WPT efficiency. This comparison proves that the proposed WPT systems, using spiral-strips DGS resonators, can 
provide higher efficiencies than that of the conventional strongly coupled printed resonators.

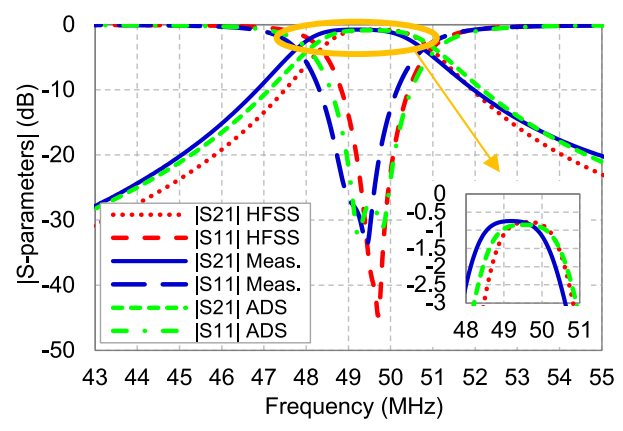

(a)

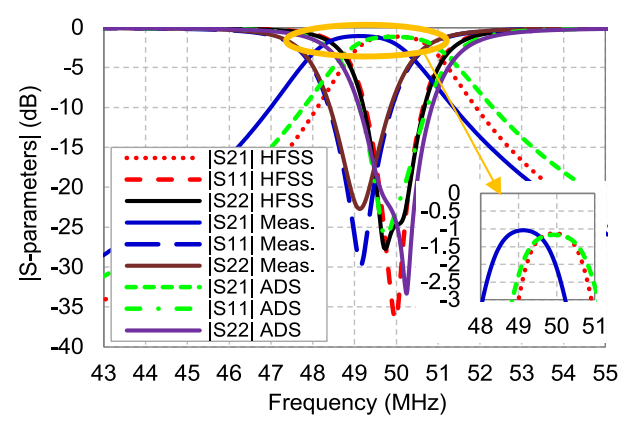

(c)

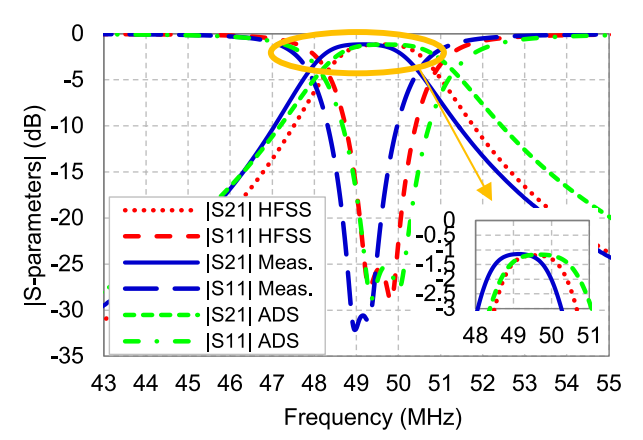

(b)

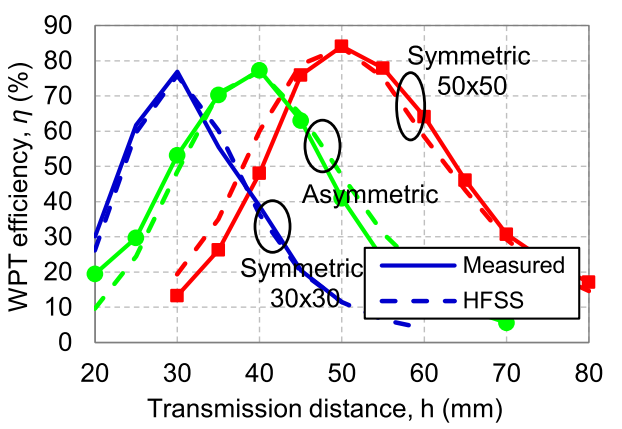

(d)

Fig. 9. Comparison between the measured and the simulated |Sparameters $\mid$ (a) Symmetric $50 \times 50 \mathrm{~mm}^{2}$ at $h=50 \mathrm{~mm}$. (b) Symmetric $30 \times 30 \mathrm{~mm}^{2}$ at $h=30 \mathrm{~mm}$. (c) Asymmetric TX $=$ $50 \times 50 \mathrm{~mm}^{2}, \mathrm{RX}=30 \times 30 \mathrm{~mm}^{2}$ at $h=40 \mathrm{~mm}$. (d) Measured and EM simulated WPT efficiency versus different transmission distances.

Table II. Comparison of the proposed WPT system with the recent published planar WPT systems

\begin{tabular}{|c|c|c|c|c|c|}
\hline Reference & $\begin{array}{c}f_{0} \\
(\mathrm{MHz})\end{array}$ & $\begin{array}{c}\mathrm{TX} \text { area } \\
\left(\mathrm{mm}^{2}\right)\end{array}$ & $\begin{array}{c}\text { RX area } \\
\left(\mathrm{mm}^{2}\right)\end{array}$ & $\begin{array}{c}\text { Distance } \\
h(\mathrm{~mm})\end{array}$ & $\begin{array}{c}\text { Efficiency } \\
\eta(\%)\end{array}$ \\
\hline [3] one layer & 13.5 & $100 \times 100$ & $100 \times 100$ & 100 & 77.27 \\
\hline [3] two layers & 13.5 & $100 \times 100$ & $100 \times 100$ & 100 & 81.67 \\
\hline [4] one layer & 13.5 & $100 \times 100$ & $100 \times 100$ & 100 & 77.27 \\
\hline [4] two layers & 13.5 & $100 \times 100$ & $100 \times 100$ & 100 & 82 \\
\hline This work, Symmetric & 50 & $50 \times 50$ & $50 \times 50$ & 50 & 84 \\
\hline This work, Asymmetric & 50 & $50 \times 50$ & $30 \times 30$ & 40 & 78 \\
\hline
\end{tabular}

\section{Conclusion}

This letter presents a new type of DGS resonators to mitigate the problems of low self-inductance that encountered by H-shape DGS resonator $[1,2]$ for a WPT system. By employing the proposed spiral-strips DGS resonator, an efficient and compact design for asymmetric WPT systems was proposed and verified by the 
design and experiment. The proposed asymmetric WPT system achieves a WPT efficiency of $78 \%$ at a transmission distance of $40 \mathrm{~mm}$. The TX and RX areas are $50 \times 50 \mathrm{~mm}^{2}$ and $30 \times 30 \mathrm{~mm}^{2}$, respectively. This system can be used in wireless charging applications for the portable electronic devices (mobile phones, laptop, etc.) that need compact RX structure irrespective of the area of the TX embedded in an external charging pad.

\section{Acknowledgments}

A part of this work is supported by a Grant-in-Aid for Scientific Research (C) (16K06301), Center for Japan-Egypt Cooperation in Science and Technology, Kyushu University, Motooka 744, Nishi-Ku, Fukuoka 819-0395, Japan, the Egyptian Ministry of Higher Education (MoHE), Cairo, Egypt, and Egypt-Japan University of Science and Technology (E-JUST), Alexandria, Egypt. 